MADPH-97-1008

\title{
CONSTRAINTS ON SEMILEPTONIC FORM-FACTORS FROM DISPERSIVE SUM RULES
}

\author{
G. BURDMAN \\ Department of Physics, University of Wisconsin, \\ Madison, WI 53706
}

\begin{abstract}
I briefly discuss the derivation of dispersive sum rules constraining semileptonic form-factors. I outline the use of these constraints in the context of charmless semileptonic decays and suggest how, in combination with other theoretical and experimental information, they may reduce the uncertainties in the extraction of $V_{u b}$.
\end{abstract}

\section{Introduction}

The recent experimental success in observing exclusive charmless semileptonic decays 1 forces us to reexamine the theoretical situation in the predictions for these decay modes. These seem to remain plagued with theoretical uncertainties. The situation is very different from the one in transitions between hadrons containing heavy quarks in both the initial and final states, where the use of heavy quark effective theory (HQET) leads to very precise predictions e.g. for $B \rightarrow D^{*} \ell \nu$ l. For heavy-to-light transitions like $B \rightarrow \pi \ell \nu, B \rightarrow \rho \ell \nu$, etc., the impact of the theoretical developments in heavy quark symmetry is less significant, and is mostly reduced to flavor symmetry relations among $B$ and $D$ decay semileptonic form-factors. There are a variety of approaches to this problem. In general, complete calculations of the form-factors are highly model-dependent. Such is the case with relativistic and non-relativistic quark models and the various QCD sum-rule calculations. On the other hand, in some cases model-independent statements can be made about these decays. Here we derive one of these model-independent results from the asymptotic behavior as dictated by perturbative QCD together with the analyticity of the form-factors 3 . As we will see, they can have a great impact in the determination of the $q^{2}$-dependence of the semileptonic form-factors, a crucial feature in predicting the rate for these modes and ultimately in extracting $V_{u b}$.

We concentrate on the $B \rightarrow \pi \ell \nu$ decay for concreteness and also simplicity. The hadronic matrix element for the $B^{0} \rightarrow \pi^{-} \ell^{+} \nu$ transition can be written

${ }^{a}$ Talk presented at the 2nd International Conference on B Physics and CP Violation, Honolulu, Hawaii, March 24-27, 1997. 
as

$$
\left\langle\pi\left(\mathbf{p}_{\pi}\right)\left|\bar{u} \gamma_{\mu} b\right| B(\mathbf{P})\right\rangle=f_{+}\left(q^{2}\right)\left(P+p_{\pi}\right)_{\mu}+f_{-}\left(q^{2}\right)\left(P-p_{\pi}\right)_{\mu}
$$

where $q^{2}=\left(P-p_{\pi}\right)^{2}$ is the momentum transferred to the leptons. In the approximation where the leptons are massless, only the form-factor $f_{+}\left(q^{2}\right)$ enters the in partial rate. This form-factor obeys a dispersion relation of the form

$$
f_{+}(t)=\frac{-\gamma}{\left(m_{B^{*}}^{2}-t\right)}+\frac{1}{\pi} \int_{s_{0}}^{\infty} \frac{\operatorname{Im}\left[f_{+}(s)\right] d s}{(s-t-i \epsilon)}
$$

where $s_{0}=\left(m_{B}+m_{\pi}\right)^{2}$. The isolated pole at $m_{H^{*}}<s_{0}$ is actually present in the $H=B$ case, whereas for the $D \rightarrow \pi$ transition the $D^{*}$ pole is located almost exactly at threshold. Although not strictly necessary for our results to be derived, it is instructive to characterize the different contributions to the imaginary part in (2). These are all possible intermediate states coupling to $B \pi$ and annihilated by the weak vertex, including the multi-particle continuum as well as resonances. These must be radial excitations of the $B^{*}$ in order to couple to this current and therefore are expected to be separated from the ground state by a typical hadronic scale of approximately $\simeq 1 \mathrm{GeV}$. Thus we expect the continuum to dominate the region just above the threshold $s_{0}$, while the resonances take over at a typical hadronic scale above $s_{0}$. This picture can be expressed by

$$
f_{+}(t)=\frac{-\gamma}{\left(m_{B^{*}}^{2}-t\right)}+\frac{1}{\pi} \int_{s_{0}}^{\Lambda^{2}} \frac{\operatorname{Im}\left[f_{+}^{\text {cont. }}(s)\right] d s}{(s-t-i \epsilon)}+\sum_{i} a_{i} \mathcal{R}_{i}(t)
$$

where the cutoff in the integral corresponds to the approximate value of $s$ where resonances start dominating the imaginary part. The function $\mathcal{R}_{i}(t)$ defined in (3) is generally given by

$$
\mathcal{R}_{i}(t)=\frac{1}{\pi}\left(\frac{\pi}{2}-\arctan \frac{s_{0}-M_{i}^{2}}{M_{i} \Gamma_{i}}\right) \frac{\left(M_{i}^{2}-t\right)}{\left(M_{i}^{2}-t\right)^{2}+M_{i}^{2} \Gamma_{i}^{2}},
$$

whereas it takes the simpler form $\mathcal{R}_{i}(t)=\left(M_{i}^{2}-t\right)^{-1}$ in the narrow width approximation. It has been common practice in the literature to approximate $f_{+}(t)$ by the first term in (2) and (3). This approximation, nearest singularity dominance, is justified in some cases. For instance, for values of $t$ very close to $m_{B^{*}}$ the first term will clearly dominate. In the case of the $B \rightarrow \pi$ transition, this is formalized in the context of chiral perturbation theory for heavy hadrons $(\mathrm{CPTHH})$ t: the $B^{*}$-pole is the leading contribution in the double (heavy quark plus chiral) expansion, which implies that $f_{+}(t)$ is given by the first term in 
(2),

$$
f_{+}(t) \approx\left(\frac{g f_{B} m_{B}^{2}}{f_{\pi}}\right) \frac{1}{m_{B^{*}}^{2}-t},
$$

where the constant $\gamma$ is now given in terms of the $B$ meson decay constant $f_{B}$ and the $\left(H^{*}-H-\pi\right)$ coupling $g$. However, this expression for the form-factor is only valid for low values of the pion recoil momentum, where the chiral expansion is justified. The $B^{*}$-pole approximation is not to be trusted for pion momenta above a typical hadronic scale of $\left(m_{\rho}-1 \mathrm{GeV}\right)$. Extrapolating the validity of this approximation is particularly dangerous in $B \rightarrow \pi \ell \nu$, where most of the rate tends to be at large recoil due to the presence of the $\left|p_{\pi}\right|^{3}$ factor.

\section{Asymptotic Behavior and Dispersive Sum Rules}

The analytic function $f_{+}(t)$ not only describes the process $B \rightarrow \pi \ell \nu$ but also processes like s-channel $\ell \nu \rightarrow B \pi$ and the t-channel $\ell B \rightarrow \nu \pi$. Moreover, its behavior for very large values of $|t|$ can be reliably estimated in perturbative QCD for exclusive processes (pQCD) . In this approach the hadronic matrix element is described by the hard scattering transition amplitude folded into an overlap integral between the two hadron state wave-functions. To leading order, the hard scattering amplitude is approximated by the one-gluon exchange diagrams. The gluon momentum satisfies

$$
Q^{2}=(1-x)^{2} m_{B}^{2}+(1-y)^{2} m_{\pi}^{2} \pm 2(1-x)(1-y) P \cdot p_{\pi}
$$

where $x$ and $y$ are the momentum fractions of the non-spectator quarks in the initial and final hadron, respectively. The positive sign in the last term in (6) corresponds to the s-channel process $\ell \nu \rightarrow B \pi$, whereas the negative sign corresponds to the t-channel, e.g. $\ell B \rightarrow \nu \pi$ as well as to the $B$ decay. In order for pQCD to be safely applicable we need $Q^{2} \gg 1 \mathrm{GeV}^{2}$. However, the wavefunction of a meson containing a heavy quark peaks at $x \simeq(1-\epsilon)$, with $\epsilon \simeq$ $\mathcal{O}\left(\Lambda_{\mathrm{QCD}} / m_{b}\right)$. This implies that in the physical region for the decay $B \rightarrow \pi \ell \nu$ the gluon momentum is $Q^{2} \lesssim 1 \mathrm{GeV}^{2}$, with the exception of a negligible high$Q^{2}$ tail of the wave-function. This casts a serious shadow over the applicability of the one-gluon exchange approximation in computing $f_{+}(t)$ in the physical region for the semileptonic decay, signaling possible large corrections.

On the other hand, outside the physical region and for large enough values of $|t|$, the condition $Q^{2} \gg 1 \mathrm{GeV}^{2}$ is satisfied. In these two regions, for $t \ll 0$ or for $t \gg M^{2}$ with $M$ the typical mass of heavy resonances, pQCD should yield a very good approximation to the form-factor, especially to its shape. This knowledge of $f_{+}(t)$ outside the physical region can be used as a boundary 
condition and connected to the decay region by using the dispersion relation (2). For this purpose, it is convenient to rewrite it as

$$
\begin{aligned}
f_{+}(t) & =-\frac{\gamma}{\left(m_{B^{*}}^{2}-t\right)}+\frac{1}{\pi} \int_{s_{0}}^{\Lambda^{2}} \frac{\operatorname{Im}\left[f_{+}^{\text {cont. }}(s)\right] d s}{s-t-i \epsilon}+\frac{1}{\pi} \int_{\Lambda^{2}}^{\Lambda^{\prime 2}} \frac{\operatorname{Im}\left[f_{+}(s)\right] d s}{s-t-i \epsilon} \\
& +\frac{1}{\pi} \int_{\Lambda^{\prime 2}}^{\infty} \frac{\operatorname{Im}\left[f_{+}(s)\right] d s}{s-t-i \epsilon} .
\end{aligned}
$$

In (7), the scale $\Lambda^{\prime}$ defines the end of the resonance region and the beginning of the perturbative regime. For sufficiently large $\Lambda^{\prime}$ the last term can be computed in pQCD. For very large values of $|t|$, for instance $t \ll 0$, the asymptotic behavior of (7) implies

$$
\begin{aligned}
f_{+}(t) \longrightarrow \frac{-1}{t} \times & \left\{-\gamma+\frac{1}{\pi} \int_{s_{0}}^{\Lambda^{2}} \operatorname{Im}\left[f_{+}^{\text {cont. }}(s)\right] d s\right. \\
& \left.+\frac{1}{\pi} \int_{\Lambda^{2}}^{\Lambda^{\prime 2}} \operatorname{Im}\left[f_{+}(s)\right] d s+p_{1}\left(t, \Lambda^{\prime}\right)\right\}
\end{aligned}
$$

where the last term in the brackets in (8) is the leading asymptotic contribution from the last term in (7). But in this limit we have $f_{+}(t) \longrightarrow f_{+}^{\mathrm{pQCD}}(t)$. The non-perturbative contributions in (8) are, individually, much larger than $f_{+}^{\mathrm{pQCD}}(t)$, which is of order $\left(\alpha_{s} / \pi\right)$. Therefore, because $f_{+}^{\mathrm{pQCD}}(t)$ is a reliable approximation to the form-factor for $t \rightarrow-\infty$, there must be large cancellations among the non-perturbative contributions. This leads to a convergence condition or dispersive sum rule of the form:

$$
\gamma-\frac{1}{\pi} \int_{s_{0}}^{\Lambda^{2}} \operatorname{Im}\left[f_{+}^{\text {cont. }}(s)\right] d s-\frac{1}{\pi} \int_{\Lambda^{2}}^{\Lambda^{\prime 2}} \operatorname{Im}\left[f_{+}(s)\right] d s \simeq 0
$$

where the equality corresponds to $f_{+}^{\mathrm{pQCD}}(t)=-p_{1}\left(t, \Lambda^{\prime}\right) / t$. 6 With this identification, corrections to (9) are of order $\left(\alpha_{s} / \pi\right)^{2}$. This sum rule translates our knowledge of the asymptotic behavior of $f_{+}(t)$ into a constraint on the non-perturbative contributions, which in turn dominate $f_{+}(t)$ in the physical region. Similar sum rules can be obtained for the other form-factors involved in weak decays. The important lesson is that the known asymptotic behavior implies a relation among the various non-perturbative components that make up the transition form-factor. This type of relations, as we will see below, might result in large deviations from the $B^{*}$-pole behavior of $f_{+}(t)$.

${ }^{b}$ The dependence on the scale $\Lambda^{\prime}$ reflects the typical scale dependence of $p Q C D$ calculations. 


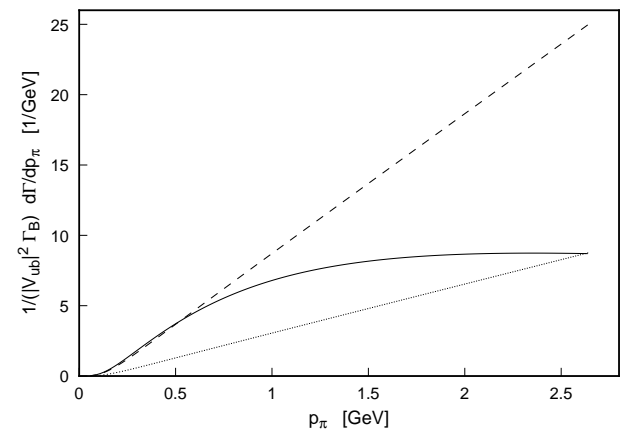

Figure 1: Pion-momentum distributions for $B^{0} \rightarrow \pi^{-} \ell^{+} \nu$. See text for captions.

\section{Consequences for $B \rightarrow$ (light) $\ell \nu$ Decays}

In order to have an idea of the impact of the dispersive sum rules on our understanding of the weak form-factors, let us write (9) in the narrow width approximation for the resonances,

$$
\gamma-c-\sum_{i=1} a_{i} \simeq 0,
$$

where $c$ is the integral above threshold of the continuum contribution and the constants $a_{i}$ are analogous to $\gamma$ but for the radially excited states of the $B^{*}$. First of all, it is not possible to have a large number of resonances contributing significantly to (10). The $a_{i}$ 's are proportional to the couplings between the excited state and the ground state $\left(B, B^{*}\right)$ and a pion. But the Adler-Weisberger sum rule 1 constraints the sum of the squares of all the couplings of excited states (radial and orbital) to be essentially 1. Either we have lots of resonances weakly coupled or only a few with couplings similar to $g$. For instance as an exercise one can construct a simple model with two radial excitations 3 . Here it is enough to use masses as computed in some successful potential model given that the intrinsic uncertainties in the spectra derived from these calculations will not be of significance in our results. Assuming typical values for the constants $f_{B}$ and $g$, making use of the dispersive sum rule leaves us with one unknown coupling which we fix by fitting to the $D^{0} \rightarrow \pi^{-} \ell^{+} \nu$ branching ratio. The result is the solid line of Figure 1. For comparison, the pure $B^{*}$-pole prediction is shown in the dashed line as normalized by CPTHH (5). Also, shown by the dotted line, is the prediction of the BSW model $\mathrm{B}$. We see that at low pion momentum the model coincides with the $B^{*}$-pole as required 
by CPTHH. However, at higher recoils the spectrum softens considerably due to the imposition of the dispersive sum rule (10), which forces the form-factor to behave as dictated by perturbative QCD at very large $|t|$. In fact, if one computes $f_{+}^{\mathrm{pQCD}}(t)$ one sees that for $t \ll 0$, but still $|t|<m_{B}^{2} \ln \left(m_{B}^{2} / A_{\mathrm{QCD}}^{2}\right)$, the shape of form-factor is given by a dipole rather than a monopoled. The suppression with respect to the $B^{*}$-pole at large $\left|p_{\pi}\right|$ forced by the sum rule gives the correct matching between the two behaviors. To see how this happens let us go back to the simple model with a few narrow resonances. The $B^{*}$-pole term of the form-factor is now modified to be ${ }^{3}$

$$
\frac{\gamma}{m_{B^{*}}^{2}-t}\left(\frac{M_{i}^{2}-m_{B^{*}}^{2}}{M_{i}^{2}-t}\right) \simeq \frac{\gamma}{m_{B^{*}}^{2}-t}\left(\frac{1}{1+v \cdot p_{\pi} / \Delta_{i}}\right)
$$

where $\Delta_{i} \equiv M_{i}-m_{B}$ is the gap between the ground state and the $i$-th excitation, and provides the scale of suppression which is of about $(0.8-1) \mathrm{GeV}$, a typical hadronic scale. Therefore the resonances in the cut give the scale for the effective suppression of the $B^{*}$-pole behavior at large pion recoil. This picture also provides us with a rule of thumb for the validity of the nearestpole-dominance approximation. This will be valid as long as the hadronic recoil is small compared to the gap between the dominant resonance (e.g. the $B^{*}$ ) and the next excitation with the right quantum numbers. This is the reason why pole dominance is a good approximation in $D$ semileptonic decays as well as in $B \rightarrow D^{(*)} \ell \nu$ decays, but not in $B \rightarrow \pi \ell \nu$, where large portions of the rate come from $v \cdot p_{\pi}>\Delta_{i}$.

\section{Outlook}

Dispersive sum rules are an important constraint on model building, as the very simplistic models presented here show. Although these models are useful as long as they are a correct parametrization of the physics, more sophistication is possible and may even be required. Another step in this approach is to apply it to other charmless decays, e.g. $B \rightarrow \rho \ell \nu$, and build similar models constrained by them that can be tested against experiment. The presence of a vector meson in the final state implies that there are now five independent form-factors, although only four are relevant if $m_{\ell}=0$ Recently new relations among heavyto-light form-factors have been derived 9 . In principle these relations can be used in a region of phase space that might be of interest, and in combination with the methods I described earlier yield predictions for decay modes like $B \rightarrow \rho \ell \nu 13$.

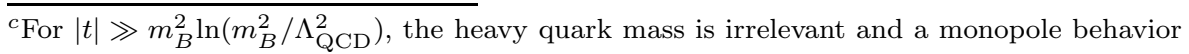
similar to that of the pion form-factor is recovered. 
Other model-independent avenues, like lattice calculations $\$ 0$ or their combina-

tion with the bounds derived from perturbative QCD dispersion relations 11.12 already place stringent constraints on the form-factors. A complete program incorporating all model-independent constraints seems to be already emerging and might result in theoretically safe predictions for the heavy-to-light form-factors and a precise determination of $V_{u b}$.

\section{Acknowledgments}

The work reported in here is based on a collaboration with J. Kambor 3 . It was supported by the U.S. Department of Energy under Grant No. DE-FG0295ER40896 and the University of Wisconsin Research Committee with funds granted by the Wisconsin Alumni Research Foundation.

\section{References}

[1] J. P. Alexander et al., Phys. Rev. Lett.77, 5000 (1996). See also R. Poling, these proceedings.

[2] M. Luke, these proceedings.

[3] G. Burdman and J. Kambor, Phys. Rev. D55, 2817 (1997).

[4] G. Burdman and J. F. Donoghue, Phys. Lett. B280, 287 (1992); M. B. Wise, Phys. Rev. D45, 2188 (1992).

[5] G. P. Lepage and S. J. Brodsky, Phys. Lett. B87, 959 (1979) and Phys. Rev. D22, 2157 (1980). For the first application to $B$ decays see A. Szczepaniak, E. M. Henley and S. J. Brodsky, Phys. Lett. B243, 287 (1990).

[6] C. A. Dominguez and N. Paver, Z. Phys. C41, 217 (1988); N. Paver and Riazuddin, Phys. Lett. B320, 364 (1994) and C. Chow and D. Pirjol, Phys. Rev. D54, 2063 (1996).

[7] E. Eichten, C. T. Hill and C. Quigg, Phys. Rev. Lett.71, 4116 (1993); FERMILAB-CONF-94/117-T and FERMILAB-CONF-94/118-T, published in the Proceeding of the CHARM2000 Workshop, Fermilab, June 1994, ed. D. Kaplan.

[8] M. Wirbel, B. Stech and M. Bauer, Z. Phys. C29, 637 (1985).

[9] B. Stech, Phys. Lett. B354, 447 (1995); J. Soares, Phys. Rev. D54, 6837 (1996).

[10] J. N. Simone, FERMILAB-CONF-96-017-T, International Symposium On Lattice Field Theory, 11-15 Jul 1995, Melbourne, Australia.

[11] C. G. Boyd, B. Grinstein and R. Lebed, Phys. Rev. Lett. 74, 4603 (1995).

[12] L. Lellouch, Nucl. Phys. B479, 353 (1996). 
[13] G. Burdman, in preparation. 